\title{
Do the Unidentified EGRET Sources Trace Annihilating Dark Matter in the Local Group?
}

\author{
J. Flix ${ }^{*}$, J.E. Taylor ${ }^{2}$, M. Martínez ${ }^{1}$, F. Prada ${ }^{3}$, J. Silk $^{2}$ and J. \\ Cortina $^{1}$ \\ ${ }^{1}$ Institut de Física d'Altes Energies, Universitat Autònoma de Barcelona, E-08193 \\ Barcelona, Spain \\ 2 Astrophysics, University of Oxford, Denys Wilkinson Building, Keble Road, \\ Oxford, OX1 3RH, UK \\ ${ }^{3}$ Instituto de Astrofísica de Andalucía (CSIC), E-18008 Granada, Spain
}

\begin{abstract}
In a cold dark matter (CDM) framework of structure formation, the dark matter haloes around galaxies assemble through successive mergers with smaller haloes. This merging process is not completely efficient, and hundreds of surviving halo cores, or subhaloes, are expected to remain in orbit within the halo of a galaxy like the Milky Way. While the dozen visible satellites of the Milky Way may trace some of these subhaloes, the majority are currently undetected. A large number of high-velocity clouds (HVCs) of neutral hydrogen are observed around the Milky Way, and it is plausible that some of the HVCs may trace subhaloes undetected in the optical. Confirming the existence of concentrations of dark matter associated with even a few of the HVCs would represent a dramatic step forward in our attempts to understand the nature of dark matter. Supersymmetric (SUSY) extensions of the Standard Model of particle physics currently suggest neutralinos as a natural well-motivated candidate for the non-baryonic dark matter of the universe. If this is indeed the case, then it may be possible to detect dark matter indirectly as it annihilates into neutrinos, photons or positrons. In particular, the centres of subhaloes might show up as point sources in gamma-ray observations. In this work we consider the possibility that some of the unidentified EGRET $\gamma$-ray sources trace annihilating neutralino dark matter in the dark substructure of the Local Group. We compare the observed positions and fluxes of both the unidentified EGRET sources and the HVCs with the positions and fluxes predicted by a model of halo substructure, to determine to what extent any of these three populations could be associated.
\end{abstract}

Keywords: Dark Matter Substructure - gamma rays: observations - HII Clouds

\section{Introduction}

Determining the nature of the unidentified EGRET sources (Hartman et al, 1999) is an important unsolved problem in modern high-energy Astrophysics. These sources constitute a major part of all the discrete sources sample detected by EGRET. Despite continuing efforts, most of them have not yet been associated with sources at other wavelengths.

* Contact e-mail address: (jflix@ifae.es)

(c) 2018 Kluwer Academic Publishers. Printed in the Netherlands. 
Identification of EGRET sources is particularly difficult because of the large uncertainties in source positions and the lack of other observations at similar wavelengths.

While a few of the unidentified EGRET sources may simply be artifacts of the Galactic diffuse emission, it is normally thought that most are genuine detections, and that some fraction may even represent a completely new population of $\gamma$-ray emitters. As the sources are located predominantly at low to mid-Galactic latitudes, it seems likely they are mainly Galactic in origin, rather than being extragalactic objects like quasars (although this may account for some faction of them - e.g. (Sowards-Emmerd et al, 2003)). Thus most of the effort to identify the EGRET sources has focused on possible Galactic counterparts such as young pulsars (Hobbs et al, 2004), supernova remnants (Torres et al, 2003), isolated neutron stars (Goldoni and Mussol, 1996), molecular clouds (Benaglia et al, 2001), objects in Gould's Belt (Gehrels et al, 2000), microquasars (Paredes, 2002), or young stellar objects like Wolf-Rayet stars or OB associations (Romero et al, 1999).

On extragalactic scales, observational cosmology has recently converged on a 'concordance' model, which appears to satisfy all or most of the observational constraints simultaneously. In this ' $\Lambda C D M$ ' cosmology, the present-day energy density of the universe is dominated by 'dark energy', possibly in the form of a cosmological constant $\Lambda$, while the remaining matter content is constrained by nucleosynthesis and the microwave background to be predominantly non-baryonic cold dark matter (CDM) (Spergel et al, 2003). The nature of this dark matter is still unknown, and its identification is currently a top priority for theoretical and experimental Astrophysics.

Weakly Interacting Massive Particles (WIMPs), non-relativistic relics from the early universe, have been proposed as one good candidate for non-baryonic dark matter. Although none of the particles in the Standard Model satisfy the requirements of being weakly interacting, massive and stable enough to be suitable relic particle, supersymmetric (SUSY) extensions of the Standard Model do provide a candidate that satisfies these requirements: the lightest SUSY particle (LSP), which ends up being one of the neutralinos $(\chi)$ in most of the SUSY-breaking scenarios. The neutralino is a majorana particle and can annihilate with itself in pairs, producing high-energy neutrinos, positrons, antiprotons and $\gamma$-rays (Bergstrom et al, 2001). Thus in principle it can be detected through high-energy observations.

In this paper we will discuss about the possibility that $\gamma$-ray emission from $\chi$ self-annihilation, occurring in dark matter subhaloes in the halo of our Galaxy, may be responsible for some of the unidentified EGRET sources. We consider the spatial distribution and fluxes of these sources, 
and compare them with the predictions of a model of halo substructure. We will also compare the source positions with the high-velocity clouds (HVCs), a population of neutral hydrogen clouds that may also trace dark matter concentrations in the galactic halo.

\section{Dark Matter Substructure in Galaxy Haloes}

In CDM cosmologies, galaxies form within dense concentrations of dark matter, called 'haloes' by analogy with the stellar halo of the Milky Way. Dark matter haloes are assembled through a process of accretion and the hierarchical merging of smaller subunits (cf. (Taylor et al, 2003) and references therein). High-resolution numerical simulations of CDM structure formation have shown that this merging process is not completely efficient; they predict that hundreds of dense halo cores, or subhaloes, should remain in orbit around present-day galaxies (e.g. (Klypin et al, 1999)). While some of these subhaloes may correspond to the few dozen satellites which surround the Milky Way Galaxy and our neighbour M31 (Mateo, 1998), the expected number of satellites exceeds the number observed by approximately an order of magnitude (Klypin et al, 1999) (Moore et al, 1999).

The current census of dwarf satellites is probably still incomplete. The local dwarf galaxies are often faint, low-brightness objects, and thus very difficult to detect, as evidenced by several discoveries made only recently (e.g. the Canis Major dwarf, (Martin et al, 2004)). Nonetheless, it seems unlikely that most of the subhaloes predicted to orbit the Milky Way will ever be detectable by stellar emission alone.

\subsection{Subhaloes as $\gamma$-Ray Sources}

The specific properties of the dark matter particle may provide an alternate means of detecting local substructure. Neutralino annihilation can generate continuum $\gamma$-ray emission, via the process $\chi \chi \rightarrow q \bar{q}$. The subsequent decay of $\pi^{0}$-mesons created in the resulting quark jets produces a continuum of $\gamma$-rays. Given a neutralino density profile $\rho_{\mathrm{dm}}(r)$ (i.e. the dark matter density profile within a subhalo), the expected annihilation $\gamma$-ray flux above an energy $E_{0}$ arriving to Earth is given by:

$$
\begin{aligned}
\Phi_{\gamma}\left(E>E_{0}\right) & =\frac{1}{4 \pi} \frac{N_{\gamma}\langle\sigma v\rangle_{\mathrm{ann}}}{2 m_{\chi}^{2}} \cdot J(\Psi) \\
J(\Psi) & =\int_{\text {l.o.s }} \rho_{\mathrm{dm}}^{2}(r) d l
\end{aligned}
$$


where $N_{\gamma}$ is the number of continuum gammas emitted per neutralino annihilation of $m_{\chi}$ mass above an energy $E_{0}$, and $\langle\sigma v\rangle_{\text {ann }}$ the averaged product of annihilation cross-section and relative velocities. The first part of Equation 1 can be evaluated for any given SUSY scenario, although the result varies by several orders of magnitude over the span of SUSY parameter space (Prada et al, 2004).

All the information about the dark matter distribution is contained in the $J(\Psi)$ term. Cosmological simulations indicate that dark matter haloes are approximately spherical, with a cuspy central density profile that scales as $\rho_{\mathrm{dm}}(r) \propto r^{-\alpha}$. The central slope of the density profile is thought to lie somewhere between $\alpha=1$ (Navarro et al, 2004) (the 'NFW profile') and $\alpha=1.5$ (Moore et al, 1998), although recent simulations generally find that central slopes are shallower than 1.5.

This profile should characterise both the main halo within which the Milky Way resides, and the subhalo cores that survive within it. The central cusp of the main halo may be affected by subsequent stages in galaxy formation, however, since baryons dominate the gravitational potential within the luminous part of bright galaxies. In particular, processes like starbursts, galaxy mergers or bar formation could heat and disrupt the dark matter cusp in the centre of the Milky Way, M31 or the brighter members of the Local Group. Smaller subhaloes should be less strongly affected by baryonic processes, and thus they may preserve 'pristine' dark matter cores, where the annihilation rate is still relatively high. Thus we will focus on comparing EGRET sources with small subhaloes, and ignore $\gamma$-ray emission (or lack thereof) from the brightest members of the Local Group.

Using the semi-analytic model described in (Taylor et al, 2003), we have generated a number of realisations of halo substructure for a galaxy like the Milky Way. For each subhalo surviving at the present day, we calculated the 'flux multiplier' $J(\Psi)$ and the relative flux, as measured by an observer $8.5 \mathrm{kpc}$ from the centre of the galaxy. A cut on subhalo masses of $>5 \cdot 10^{7} M_{\odot}$ was applied, as the models are incomplete below this mass limit.

In Figure 1 (left) we compare the predicted fluxes from the subhaloes in the each of our model systems with the fluxes measured for the unidentified EGRET sources. For each realisation, we have scaled the luminosity of the brightest subhalo to the luminosity of the brightest EGRET source. This gives an upper limit on the emissivity of the dark matter, for that particular model halo. By comparing the cumulative luminosity functions, we can also determine how many of the unidentified sources could be subhaloes, assuming this scaling and a luminosity function like that of the model halo. This upper limit is between 10 and 58 of the EGRET sources, depending on the realisation, with a 

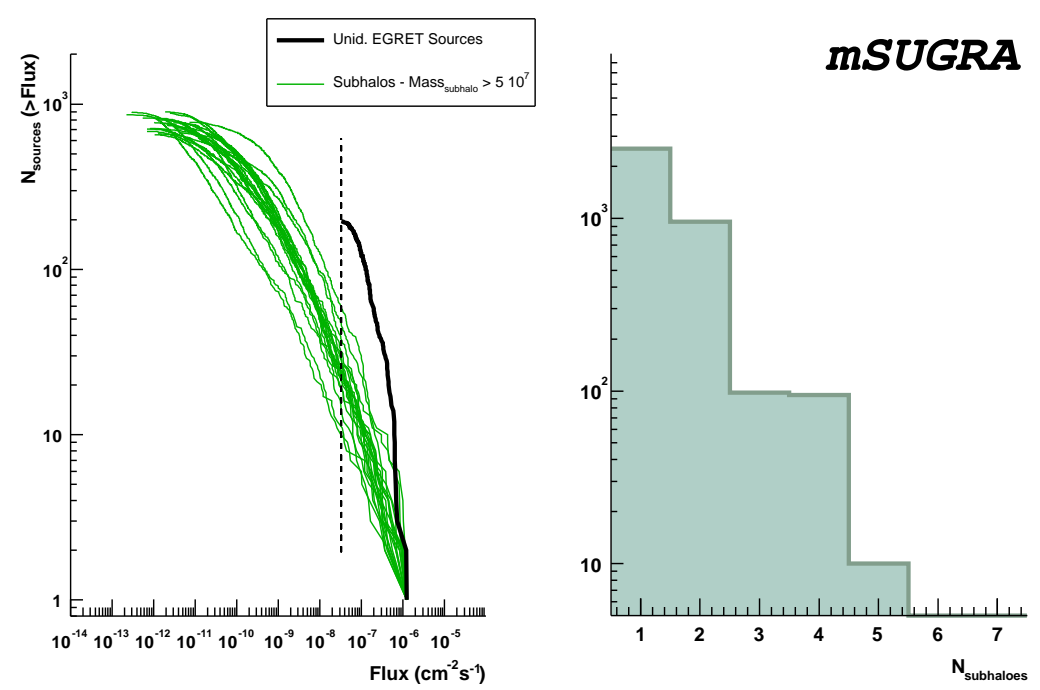

Figure 1. (left) Constrains to the maximum number of subhaloes compatible to unidentified EGRET sources. (right) Number distribution of all subhaloes with fluxes detectable by EGRET in a mSUGRA scenario.

mean value of $26 \pm 11$. If we consider a neutralino of with $m_{\chi}=100$ $\mathrm{GeV}$, we can compute the number of gammas above the EGRET energy threshold with the Darksusy package (Gondolo et al, 2004). The $\langle\sigma v\rangle_{\text {ann }}$ needed to achieve such fluxes are from $1 \cdot 10^{-25}$ to $5 \cdot 10^{-24} \mathrm{~cm}^{3} \mathrm{~s}^{-1}$. These values exceed by one or two orders of magnitude the allowed WMAP $\langle\sigma v\rangle_{\text {ann }}$ in some SUSY scenarios. These numbers constrain an upper bound on the $\langle\sigma v\rangle_{\text {ann }}$ for a $100 \mathrm{GeV}$ neutralino.

As an example for a particular SUSY model, we consider to the set of mSUGRA scenarios discussed in (Prada et al, 2004). The range of models satisfying the WMAP relic density limits correspond to $\chi$ masses between approximately $70 \mathrm{GeV}$ and $1400 \mathrm{GeV}$, while $\langle\sigma v\rangle_{\text {ann }}$ lies in the range $1 \cdot 10^{-29}$ to $3 \cdot 10^{-26} \mathrm{~cm}^{3} \mathrm{~s}^{-1}$. For these SUSY models, we compute the expected $\gamma$-ray flux above $100 \mathrm{GeV}$ for all simulated subhaloes. The Figure 1(right) shows the number distribution of all subhaloes with fluxes detectable by EGRET in this mSUGRA scenario. The number of detectable subhaloes vary from 1 to 5 . Most favourable models correspond to those neutralinos with $\langle\sigma v\rangle_{\text {ann }}$ ranging between $3 \cdot 10^{-26} \mathrm{~cm}^{3} \mathrm{~s}^{-1}$ and $10^{-27} \mathrm{~cm}^{3} \mathrm{~s}^{-1}$ with masses from 100 to $400 \mathrm{GeV}$.

\section{Comparison with a Different Population of Halo Objects}

Given the small number of possible matches to between subhaloes and EGRET sources, it would be very useful to find another observable 
tracer of halo substructure. The High-Velocity Clouds (HVCs) are isolated concentrations of neutral hydrogen whose velocities distinguish them from the gas in the galactic disk (Blitz et al, 1999). The kinematics of these clouds suggest that they may orbit the Milky Way at large distances, or that they may even be distributed throughout the Local Group. While the Compact HVCs (CHVCs) seem the most promising tracers of dark substructure (Braun and Burton, 1999), even the less compact clouds show similarities to subhaloes in their distribution, once cuts in halo distance are applied (Putman and Moore, 2001). The detection of a tidal interaction between a non-compact HVC and the dwarf galaxy LGS 3 further supports this association (Robishaw et al, 2002). Thus we will compare the distribution of both types of clouds with that of our model subhaloes, and with the distribution of EGRET sources.

\subsection{An All-sky Catalogue of High Velocity Clouds}

Two different catalogues of High Velocity Clouds are available for the two hemispheres, and they can be combined to form an all-sky catalogue of HVCs. The northern LDS survey (Heij et al, 2002) covered the sky north of declination $-30^{0}$, while southern HIPASS survey (Putman et al, 2002) covered the sky south declination $+2^{0}$. Both surveys are different in terms of sensitivity, angular resolution, velocity coverage and velocity resolution, but the same selection criteria was applied to both to identify isolated hydrogen clouds (Heij et al, 2002). In Table 3.1 we list the main parameters of each catalogue. Approximate positional accuracies were estimated by comparing published values for known dwarf galaxies with the positions of the associated hydrogen clouds.

Table I. Observational properties for HIPASS and LDS HVCs surveys.

\begin{tabular}{ccc}
\hline & LDS Survey & HIPASS Survey \\
\hline$V_{\text {LSR coverage }}$ & $-450 \mathrm{kms}^{-1}$ to $+400 \mathrm{kms}^{-1}$ & $-700 \mathrm{kms}^{-1}$ to $+500 \mathrm{kms}^{-1}$ \\
V resolution & $1.0 \mathrm{kms}^{-1}$ & $26.4 \mathrm{kms}^{-1}$ \\
$5 \sigma$ T Sensitivity & $70 \mathrm{mK}$ & $1 \mathrm{mK}$ \\
Angular resolution & 36 & 15.5 \\
Positional accuracy & 15 & 10 \\
\hline
\end{tabular}

A total of 917 clouds were catalogued in the northern LDS survey, of which 777 are HVCs, 90 are CHVCs (isolated clouds in position and velocity), 37 were designated as CHVC: and 13 designated as CHVC? (clouds which were not unambiguously designated as CHVCs by the 


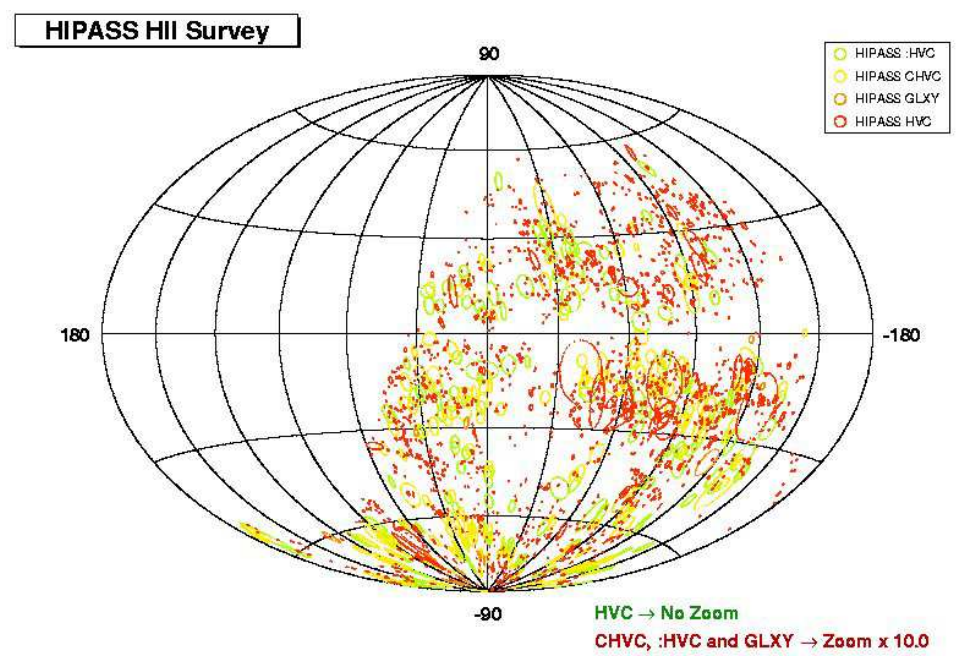

Figure 2. Hydrogen clouds included in HIPASS survey.

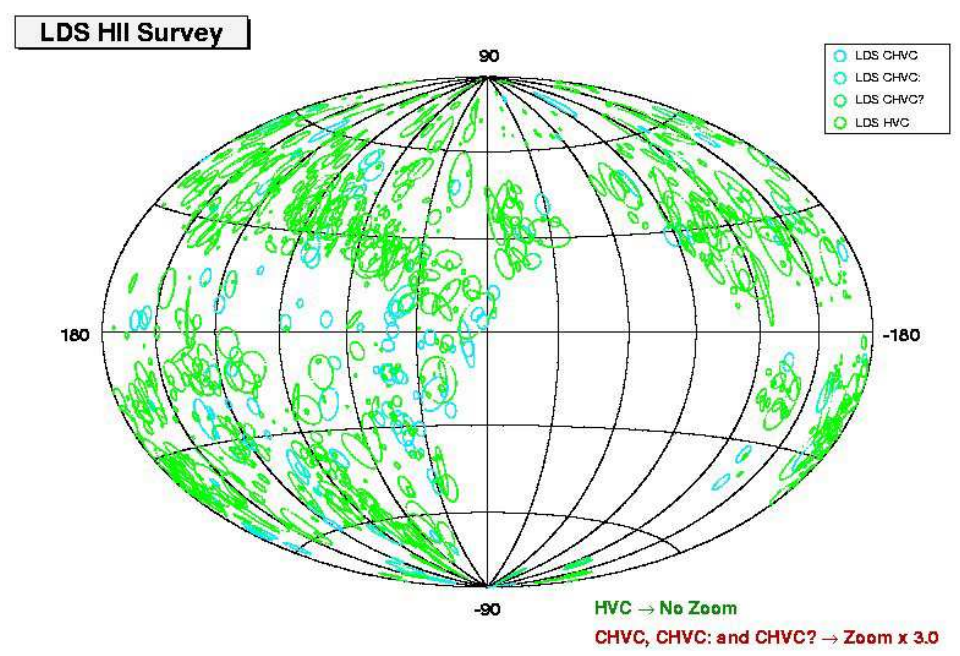

Figure 3. Hydrogen clouds included in LDS survey.

criteria described in (Putman et al, 2002)). For the HIPASS southern survey, a total of 1997 clouds were detected, of which 41 are actually galaxies (GLXY), 1618 are HVCs, 179 are CHVCs and 159 are designated as :HVC as they could not be unambiguously classified as CHVCs because their elongation towards low level extended emissions. All the hydrogen clouds detected by either survey are shown in Figure 2 and Figure 3. 


\subsection{Positional Coincidences Between HVCs and UNIDENTIFIED EGRET SOURCES}

Given the HVC positions from the HIPASS and LDS catalogues, we can test for positional coincidence between hydrogen clouds and $\gamma$ ray sources. More specifically, we can compare the positions of the maximum column density in neutral hydrogen for each cloud with the positions of the unidentified EGRET sources, on the supposition that both trace the dynamical centres of bound dark matter subhaloes. In order to determine the significance of positional coincidences, we define a relative distance between each hydrogen cloud and the each of the unidentified EGRET sources as:

$$
\delta^{2}=\frac{\left|\vec{x}_{\text {egret }}-\vec{x}_{\text {cloud }}\right|^{2}}{\sigma_{\text {egret }}^{2}+\sigma_{\text {cloud }}^{2}}
$$

where $\vec{x}_{\text {egret }}$ and $\vec{x}_{\text {cloud }}$ are the galactic longitude and latitude of the unidentified EGRET sources and the hydrogen clouds, expressed in degrees. We take as an characteristic uncertainty in the EGRET positions $\sigma_{\text {egret }}=\theta_{95} / 2$, where $\theta_{95}$ is the $95 \%$ EGRET detection confidence contour. For $\sigma_{\text {cloud }}$ we choose the positional accuracies of HIPASS and LDS surveys given above. As $\sigma_{\text {egret }}>\sigma_{\text {cloud }}, \delta$ values will be dominated by $\sigma_{\text {egret }}$.

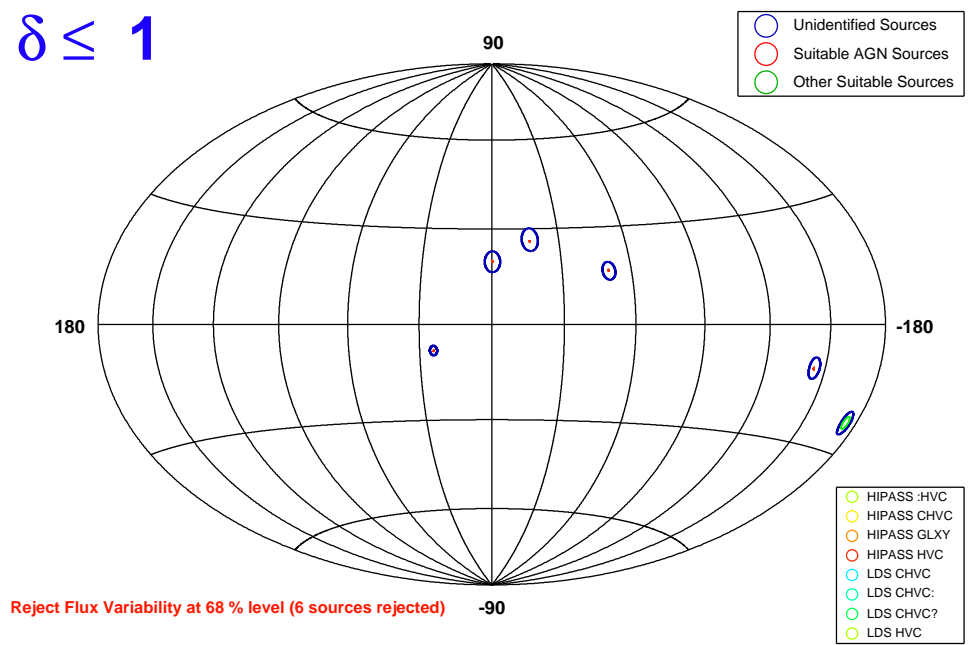

Figure 4. Unidentified EGRET sources whose positions coincide with HVCs at $\delta \leq 1$, after applying $68 \%$ flux variability rejection.

A total of 10 HIPASS HVCs and 2 LDS HVCs have an unidentified EGRET source lying within $\delta \leq 1$ of their point of maximum emission. 
As the number of unidentified EGRET sources in the field of view of HIPASS(LDS) surveys are quite similar, the different number of coincidences found for the two catalogues may be explained because the better sensitivity of HIPASS survey (HIPASS contains roughly double HI clouds than LDS) and worst LDS positional accuracies.

The $\gamma$-ray emission from neutralino annihilation should be steady on observationally accessible timescales. The variability of all sources in the 3rd EGRET Catalogue has been evaluated, assuming a model of the flux distribution to characterise the flux variation, and using both positive flux detections and upper limits (Nolan et al, 2003). This method is quite successful at distinguishing between between the known (variable) quasars and the known (non-variable) pulsars. As we are only interested in those steady $\gamma$-ray emitters, independent of how much variable they are, we make use of the $V_{12}$ parameter, which indicates the confidence with which the hypothesis of variability can be rejected for a given source $(100 \%=$ steady source). If we apply a cut at the $68 \%$ level to our $\delta \leq 1$ sample, 6 sources are rejected, resulting in 5 (1) remaining coincidences between HVCs to and unidentified EGRET sources for the HIPASS (LDS) surveys. These sources are shown in Figure 4 and listed in Table 3.2.

To check for significance of these coincidences, we perform several simulations in which we distribute $10^{4}$ fake EGRET sources isotropically and evaluate the probability of finding coincident HVCs and EGRET sources, after including the rejection factor from variability. The average number of random associations found were $1.0 \pm 0.3$ for LDS survey and $3.1 \pm 0.5$ for HIPASS survey, for the same coincidence cut criteria of $\delta \leq 1$. Thus the real result obtained for HIPASS clouds is only marginally $(4 \sigma)$ above the level expected from random coincidence.

Table II. Unidentified EGRET Sources and HVCs Found to be Spatially Coincident. $\dagger=$ HIPASS Survey, $\ddagger=$ LDS Survey.

\begin{tabular}{ccc}
\hline 3rd EGRET Name & HII Name & $\sigma$ \\
\hline $\mathrm{J} 0439+1105$ & HVC $186.2-22.8-206 \ddagger$ & $\mathbf{0 . 1 8}$ \\
$\mathrm{J} 0616-0720$ & HVC $215.8-11.2+162 \dagger$ & $\mathbf{0 . 5 4}$ \\
$\mathrm{J} 1329-4602$ & HVC $310.0+16.5+182 \dagger$ & $\mathbf{0 . 5 1}$ \\
$\mathrm{J} 1527-2358$ & HVC $343.1+25.9-144 \dagger$ & $\mathbf{0 . 9 8}$ \\
$\mathrm{J} 1635-1751$ & HVC $359.6+19.7-149 \dagger$ & $\mathbf{0 . 3 2}$ \\
$\mathrm{J} 1904-1124$ & HVC 024.1-08.2-238 $\dagger$ & $\mathbf{0 . 4 8}$ \\
\hline
\end{tabular}




\section{Conclusions}

If the dark matter particle is a supersymmetric neutralino, one of the most popular candidates currently under consideration, then local concentrations of dark matter may be detectable as $\gamma$-ray sources distributed throughout the halo of the Milky Way. In this paper, we have compared the luminosities of unidentified sources in the EGRET $\gamma$-ray catalogue with the luminosities of local dark matter substructure predicted by a semi-analytic model of neutralino annihilation in the halo of the Milky Way. We have also looked for spatial coincidences between EGRET sources and high-velocity neutral gas, which could be an observational tracer of local dark matter.

We find that most of the unidentified EGRET sources cannot be dark matter subhaloes. Whatever the emissivity of annihilating dark matter, the cumulative luminosity function for the two populations is sufficiently different that only $\sim 26 \pm 11$ of the EGRET sources could correspond to local substructure. Applying further cuts to sources on the basis of location or variability should place an even stronger constraint on the number of possible sources, and thus on the properties of dark matter.

We also find that most of the HVCs cannot correspond to EGRET sources either. While there are a few close positional coincidences between the two populations, the overall number is roughly that expected from random superposition. Thus there is no indication that the EGRET sources are associated with HVCs, whether or not the latter trace dark matter. This is interesting, because HVCs could also produce $\gamma$-rays through other, less exotic mechanisms, such as interactions with cosmic rays. Comparing HVCs with sources in lower energy catalogues might produce a stronger constraint on this mechanism, however, since the spectrum of $\gamma$-rays produced in this case is broader than that expected for $\chi$ annihilation.

\section{References}

Benaglia P., et al. 2001 A\&A 366, 605.

Bergstrom L., et al. 2001 Phys. Rev. Lett. 87, 251301.

Blitz L., et al. 1999 ApJ 514, 818.

Braun R., and Burton W. B. 1999 A\&A 341, 437.

Gehrels N., et al. 2000 Nature 404, 363.

Goldoni P., and Musso C. 1996 A\&A Supp. 120, 103.

Gondolo P., et al. 2004 JCAP 407, 8.

Hartman R. C., et al. 1999 ApJ Supp. 123, 79.

Heij V. de, Braun R. and Burton W. B. 2002 A\&A 391, 159.

Heij V. de, Braun R. and Burton W. B. 2002 A\&A 392, 417. 
Hobbs G., et al. 2004 MNRAS 352, 1439.

Klypin A., et al. 1999 ApJ 522, 82.

Martin N. F., et al. 2004 MNRAS 348, 12.

Mateo M. L. 1998 Annual Review of A\&A 36, 435.

Moore B., et al. 1998 ApJ Lett. 499, L5.

Moore B., et al. 1999 ApJ Lett. 524, L19.

Navarro J. F., et al. 2004 ApJ 490, 493.

Nolan P. L., et al. 2003 ApJ 597, 615.

Paredes J. M. 2002 Mem. Soc. Astron. Ital. 73, 900.

Prada F., et al. 2004 astro-ph/0401512 (in press).

Putman M., and Moore B. 2001 ASP Conference Proceedings 273, 1952(2):155.

Putman M., et al. 2002 ApJ 123, 873.

Robishaw T., Simon J. D. and Blitz L. 2002 ApJ 580, 129.

Romero G. E., et al. 1999 A\&A 348, 868.

Sowards-Emmerd D., et al. 2003 ApJ 590, 109.

Spergel D. N., et al. 2003 ApJ Supp. 148, 175.

Taylor J. E., et al. 2003 Dark Matter in Galaxies, International Astronomical Union. Symposium no. 220, held 22-25 July, 2003 in Sydney, Australia.

Torres D. F., et al. 2003 Phys. Rep. 382, 303. 
DarkMatter_and_UNID_EGRET_v4.tex; 29/11/2018; $16: 54 ;$ p.12 